\title{
Qualidades psicométricas do Questionário de Capacidades e Dificuldades: revisão da literatura'
}

\author{
Psychometric properties of the Strengths and Difficulties \\ Questionnaire: a literature review
}

\author{
Adriana Martins SAUR ${ }^{2}$ \\ Sonia Regina LOUREIRO 3
}

\begin{abstract}
Resumo
O Questionário de Capacidades e Dificuldades (Strengths and Difficulties Questionnaire) é um instrumento amplamente pesquisado, de uso livre, que visa avaliar a saúde mental de crianças e adolescentes. Dada a relevância do uso de instrumentos aferidos para a avaliação de indicadores de saúde mental, este trabalho objetivou analisar a produção científica relacionada às propriedades psicométricas do Questionário. Procedeu-se a um levantamento bibliográfico de estudos indexados nas bases de dados MedLine, PsycINFO e Lilacs, a partir da publicação original do instrumento, abrangendo o período de 1997 a 2010, utilizando-se as seguintes palavras-chave: Strengths and Difficulties Questionnaire and psychometric properties; e Strengths and Difficulties Questionnaire and validity or reliability. Foram selecionados e analisados 51 estudos psicométricos. Os resultados indicaram índices positivos de validade e fidedignidade em 21 países, incluindo o Brasil, caracterizando seu alcance transcultural e sua aplicabilidade na área de saúde mental infanto-juvenil.
\end{abstract}

Unitermos: Comportamento. Precisão do teste. Questionário. Saúde mental. Teste de validade.

\begin{abstract}
The Strengths and Difficulties Questionnaire is a broadly investigated tool, available for use free of charge, with the aim of assessing the mental health of children and adolescents. Given the importance of using standardized instruments for assessing mental health indicators, the aim of this study was to review scientific literature related to the psychometric properties of the Strengths and Difficulties Questionnaire. A search in MedLine, PsycINFO and Lilacs databases was performed, starting from year of the original publication of the questionnaire, from 1997 to 2010, using the keywords: Strengths and Difficulties Questionnaire and psychometric properties, and Strengths and Difficulties Questionnaire and validity or reliability. Fifty-one psychometric studies were selected and analyzed. The results indicated positive levels of validity and reliability in 21 countries, including Brazil, highlighting its cross-cultural range and applicability in the area of mental health for children and adolescents.
\end{abstract}

Uniterms: Behavior. Test reliability. Questionnaire. Mental health. Test validity.

\section{$\boldsymbol{\nabla \nabla \nabla \nabla}$}

1 Artigo elaborado a partir da tese de A.M. SAUR, intitulada "Riscos biológicos e aspectos cognitivos, comportamentais e emocionais de uma coorte de escolares". Universidade de São Paulo, 2012. Apoio: Fundação de Amparo à Pesquisa do Estado de São Paulo e Conselho Nacional de Desenvolvimento Científico e Tecnológico.

2 Universidade de São Paulo, Faculdade de Filosofia, Ciências e Letras de Ribeirão Preto, Programa de Pós-Graduação, Departamento de Psicologia. Av. dos Bandeirantes, 3900, Monte Alegre, 14040-901, Ribeirão Preto, SP, Brasil. Correspondência para/Correspondence to: A.M. SAUR. E-mail: <adrianasaur@yahoo.com.br>.

3 Universidade de São Paulo, Faculdade de Medicina de Ribeirão Preto, Departamento de Neurociências e Ciências do Comportamento. Ribeirão Preto, SP, Brasil. 
Os problemas de saúde mental na infância e na adolescência são comuns e podem se manifestar por diversas dificuldades, tais como as comportamentais, emocionais, sociais e de rendimento escolar, prejudicando o desenvolvimento e o aproveitamento dos recursos potenciais. A maioria das crianças com esses problemas não recebem tratamento adequado, o que pode favorecer a ocorrência de diversos eventos graves na vida adulta (Fleitlich-Bilyk \& Goodman, 2004).

Durante várias décadas foram propostos e utilizados vários questionários para a avaliação dos indicadores de psicopatologia de crianças e adolescentes, como os questionários de Rutter (Rutter, 1967) e de Achenbach (Achenbach, 1991). Entretanto, apesar de muito úteis, estes questionários apresentam como principal limitação a sua grande extensão. Inspirado por estas questões, Robert Goodman propôs em 1997 o Strengths and Difficulties Questionnaire (SDQ) ou Questionário de Capacidades e Dificuldades, desenhado para ser um questionário curto e simples, clinicamente útil e com boa aceitação pelos respondentes (Fleitlich, Cortázar \& Goodman, 2000; Goodman, 1997). Trata-se de um questionário de rastreamento (screening) de problemas de saúde mental, proposto para avaliar o comportamento de crianças e adolescentes dos 4 aos 16 anos.

Desde seu desenvolvimento, o SDQ tornou-se o instrumento de pesquisa mais amplamente utilizado para a detecção de problemas relacionados à saúde mental infanto-juvenil (Vostanis, 2006), encontrando-se gratuitamente disponível em mais de 40 idiomas, incluindo o português (http://www.sdqinfo.com). É composto por 25 itens, sendo 10 itens sobre capacidades, 14 itens sobre dificuldades e um item neutro. Está dividido em cinco subescalas, cada uma com cinco afirmações, a saber: sintomas emocionais, problemas de conduta, hiperatividade, problemas de relacionamento com colegas e comportamento pró-social. O instrumento é apresentado em três versões, indicadas para serem respondidas pelas próprias crianças (acima de 11 anos), por seus pais ou responsáveis e pelos professores. As alternativas para resposta apresentam como opções falso (zero ponto para esse tipo de resposta), mais ou menos verdadeiro (um ponto) e verdadeiro (dois pontos), podendo ser assinalada apenas uma única opção por item. Para cada uma das cinco subescalas a pontuação pode variar de 0 a 10, sendo a pontuação do escore total de dificuldades gerada pela soma dos resultados de todas as subescalas, exceto a de sociabilidade, podendo variar de 0 a 40 pontos. A nota de corte para o escore total de dificuldades, estabelecida para a população da Inglaterra, local de condução dos estudos originais de padronização do SDQ, foi de 17 para a versão dos pais e 16 para a versão dos professores.

No Brasil, uma extensa discussão sobre a avaliação psicológica e a utilização de testes têm levantado questões importantes e norteadoras para o avanço do conhecimento na área (Primi, 2010). Conforme apontam Noronha, Primi e Alchieri (2004), as técnicas utilizadas na avaliação psicológica têm provocado questionamentos na comunidade científica e profissional brasileira, tanto no que se refere à qualidade dos instrumentos e ao uso que os psicólogos fazem deles, quanto em relação a seus principais parâmetros psicométricos. Nesse contexto, a utilização de instrumentos aferidos e padronizados para a população brasileira tem sido cada vez mais valorizada, ressaltando-se a importância da divulgação científica de estudos que abordem tais instrumentos e suas qualidades psicométricas.

Desta maneira, o SDQ configura-se como uma promissora alternativa dentro do cenário brasileiro, onde instrumentos padronizados para a avaliação de indicadores de saúde mental infanto-juvenil ainda são escassos. Além disso, considera-se que o conhecimento das taxas de transtornos mentais na população geral auxilia no planejamento dos serviços de saúde oferecidos à comunidade e na identificação de pessoas ou grupos em risco, servindo de base para a indicação de tratamentos e para o desenvolvimento de programas de prevenção em saúde mental.

Dada a relevância do uso de instrumentos aferidos para a avaliação da saúde mental infanto-juvenil que sejam de fácil aplicação e correção, este trabalho tem como objetivo realizar uma revisão da literatura indexada, no período de 1997 a 2010, identificando e analisando estudos sobre as propriedades psicométricas do SDQ. Desse modo, pretende-se divulgar as qualidades psicométricas do SDQ, assim como suas contribuições e limites, norteando a realização de novas pesquisas, tanto com esse instrumento como em estudos que possam tomá-lo como referência na aferição de outros instrumentos de avaliação de indicadores de saúde mental infanto-juvenil. Considera-se que, no atual cenário de 
avaliação psicológica no Brasil, a apresentação dessa revisão fornecerá à comunidade científica um conhecimento mais sistemático sobre o instrumento, amplamente utilizado no mundo e com qualidades psicométricas extensivamente estudadas.

\section{Método}

Procedeu-se a um levantamento bibliográfico nas bases de dados MedLine e PsycINFO, por meio das seguintes combinações de palavras-chaves: 1) (SDQ or Strenghts and Difficulties Questionnaire) and psychometric properties; e 2) (SDQ or Strenghts and Difficulties Questionnaire) and (validity or reliability). Para a base de dados Lilacs, utilizaram-se as correspondentes combinações na língua portuguesa, a saber: 1) (SDQ ou Questionário de Capacidades e Dificuldades) e propriedades psicométricas; e 2) (SDQ ou Questionário de Capacidades e Dificuldades) e (validade ou fidedignidade). Foram definidos como critérios de inclusão artigos psicométricos nos idiomas inglês, português e espanhol, selecionados a partir da publicação original do instrumento, abrangendo o período de 1997 a 2010, em periódicos ou revistas especializadas e indexados nas referidas bases de dados. Foram excluídos artigos cujo tema diferisse do objetivo proposto, assim como teses, dissertações, livros, capítulos de livros e estudos de revisão. Foram identificados 339 artigos e, com base na leitura de seus resumos, selecionaram-se 51 que atenderam aos critérios de inclusão. Todos os 51 artigos selecionados foram lidos em sua íntegra e analisados quanto a suas características psicométricas.

\section{Resultados e Discussão}

Para facilitar a análise, apresentar-se-ão as principais características dos estudos incluídos quanto às amostras estudadas e versões utilizadas, aos países onde os estudos foram desenvolvidos e às propriedades psicométricas abordadas.

\section{Características relacionadas à versão utilizada do SDQ e composição da amostra:}

NaTabela 1 encontram-se apresentadas informações sobre o tipo de versão do SDQ utilizada (pais, professores ou autorrelato) e sobre a composição da amostra, no que diz respeito ao tipo de amostra, número de participantes, sexo e faixa etária.

Ao se analisar a Tabela 1, verifica-se que a versão do SDQ mais utilizada é a respondida pelos pais, seguida pela versão de autorrelato, enquanto a versão menos utilizada é a respondida pelos professores, sendo expressivo o número de estudos que utilizaram as três versões.

Em relação à composição das amostras, verifica-se predomínio daquelas coletadas na comunidade, seguidas pela utilização combinada de amostra clínica e da comunidade. Poucos estudos utilizaram somente amostras clínicas, o que reforça as características de rastreador do instrumento.

Tabela 1. Principais características dos estudos analisados ( $n=51)$.

\begin{tabular}{|c|c|c|}
\hline Características & f & (\%) \\
\hline \multicolumn{3}{|l|}{ Versão do SDQ utilizada } \\
\hline Pais & 17 & 33,3 \\
\hline Professores & 1 & 2,0 \\
\hline Auto-relato & 12 & 23,5 \\
\hline Pais e Professores & 6 & 11,7 \\
\hline Pais e Auto-relato & 4 & 7,9 \\
\hline Professores e Auto-relato & - & - \\
\hline Pais, Professores e Auto-relato & 10 & 19,6 \\
\hline Auto-relato e versão computadorizada & 1 & 2,0 \\
\hline Composição da Amostra & f & $\%$ \\
\hline \multicolumn{3}{|l|}{ Tipo de amostra: } \\
\hline Amostra da comunidade & 28 & 54,9 \\
\hline Amostra clínica & 7 & 13,7 \\
\hline Amostra da comunidade e amostra clínica & 16 & 31,4 \\
\hline \multicolumn{3}{|l|}{ Número de participantes: } \\
\hline$<500$ & 11 & 21,6 \\
\hline $500-1000$ & 11 & 21,6 \\
\hline $1001-5000$ & 19 & 37,2 \\
\hline $5001-10000$ & 5 & 9,8 \\
\hline $10000-20000$ & 2 & 3,9 \\
\hline$>20000$ & 3 & 5,9 \\
\hline \multicolumn{3}{|l|}{ Sexo: } \\
\hline Ambos os sexos & 50 & 98,0 \\
\hline Meninas & 1 & 2,0 \\
\hline \multicolumn{3}{|l|}{ Faixa etária: } \\
\hline Pré-escolar (3 - 6 anos) & 1 & 2,0 \\
\hline Escolar (7 - 12 anos) & 5 & 9,8 \\
\hline Adolescente (13 - 18 anos) & 4 & 7,8 \\
\hline Pré-escolar + escolar (3 - 12 anos) & 10 & 19,6 \\
\hline Escolar + adolescente (7 - 18 anos) & 13 & 25,5 \\
\hline Pré-escolar + escolar + adolescente (3 - 18 anos) & 18 & 35,3 \\
\hline
\end{tabular}

SDQ: Strengths and Difficulties Questionnaire. 
O número de participantes dos estudos foi bastante diversificado, variando entre estudos com menos de 500 sujeitos até outros com mais de 20 mil participantes, tendo no mínimo 132 participantes e no máximo 40163 (somando-se em ambos os casos todas as amostras incluídas no estudo). A maior parte dos estudos coletaram entre 1 mil e 5 mil participantes, o que pode ser considerado bastante satisfatório para os objetivos psicométricos dos estudos analisados.

Em relação ao sexo dos participantes, somente um estudo utilizou exclusivamente meninas, enquanto todos os demais incluíram ambos os sexos.

A faixa etária dos participantes surpreende pela amplitude, uma vez que a maior parte dos estudos incluiu crianças e adolescentes dos 3 aos 18 anos, abrangendo vários períodos de desenvolvimento (pré-esco- lar, escolar e adolescência). Destaca-se que a amplitude das faixas etárias englobadas nos estudos sinaliza as diversas possibilidades de aplicação do SDQ para diferentes idades e momentos do desenvolvimento.

\section{Países de realização dos estudos}

Outro importante aspecto a ser contemplado é o local de realização dos estudos, no sentido de se obter um panorama geral sobre a amplitude do alcance do SDQ. O Anexo apresenta os 51 estudos analisados (por autor e ano), assim como os países de realização dos estudos e o tipo de propriedade psicométrica avaliada em cada país.

A Tabela 2 apresenta, de maneira sintetizada, os dados contidos no Anexo 1.

Tabela 2. Local de realização dos estudos e propriedades psicométricas analisadas $(n=51)$.

\begin{tabular}{|c|c|c|c|c|c|c|c|c|c|c|c|c|}
\hline \multirow{3}{*}{ Continente/Países } & \multicolumn{12}{|c|}{ Propriedade psicométrica } \\
\hline & \multicolumn{2}{|c|}{ V } & \multicolumn{2}{|c|}{$V+F$} & \multicolumn{2}{|c|}{$V+F+P$} & \multicolumn{2}{|c|}{$V+P$} & \multicolumn{2}{|c|}{$F+P$} & \multicolumn{2}{|c|}{ Total } \\
\hline & $f$ & $\%$ & $f$ & $\%$ & $f$ & $\%$ & $f$ & $\%$ & $f$ & $\%$ & $f$ & $\%$ \\
\hline \multicolumn{13}{|l|}{ América do Norte } \\
\hline Estados Unidos & 1 & 25,0 & 2 & 50,0 & & & & & 1 & 25,0 & 4 & 7,8 \\
\hline \multicolumn{13}{|l|}{ Europa } \\
\hline Inglaterra & 4 & 44,4 & 5 & 55,6 & & & & & & & 9 & 19,6 \\
\hline Alemanha & 1 & 16,6 & 4 & 66,7 & 1 & 16,6 & & & & & 6 & 11,7 \\
\hline Holanda & 1 & 16,6 & 4 & 66,7 & 1 & 16,6 & & & & & 6 & 11,7 \\
\hline Suécia & 1 & 20,0 & 1 & 20,0 & 3 & 60,0 & & & & & 5 & 9,8 \\
\hline Noruega & 1 & 33,3 & 1 & 33,3 & 1 & 33,3 & & & & & 3 & 5,9 \\
\hline Finlândia & & & & & 1 & 100,0 & & & & & 1 & 2,0 \\
\hline EUA e Inglaterra & & & 1 & 100,0 & & & & & & & 1 & 2,0 \\
\hline Bélgica & & & 1 & 100,0 & & & & & & & 1 & 2,0 \\
\hline Itália & & & 1 & 100,0 & & & & & & & 1 & 2,0 \\
\hline França & & & & & & & & & 1 & 100,0 & 1 & 2,0 \\
\hline Vários países da Europa & & & 1 & 100,0 & & & & & & & 1 & 2,0 \\
\hline Rússia & & & & & & & 1 & 100,0 & & & 1 & 2,0 \\
\hline \multicolumn{13}{|l|}{ África } \\
\hline Congo & & & & & 1 & 100,0 & & & & & 1 & 2,0 \\
\hline \multicolumn{13}{|l|}{ Ásia } \\
\hline China & & & 1 & 33,3 & 2 & 66,7 & & & & & 3 & 5,9 \\
\hline Yêmen & 1 & 50,0 & & & & & 1 & 50,0 & & & 2 & 3,9 \\
\hline Paquistão & & & & & & & 1 & 100,0 & & & 1 & 2,0 \\
\hline Japão & & & & & 1 & 100,0 & & & & & 1 & 2,0 \\
\hline Sri Lanka & & & & & 1 & 100,0 & & & & & 1 & 2,0 \\
\hline \multicolumn{13}{|l|}{ Oceania } \\
\hline Austrália & & & 1 & 50,0 & 1 & 50,0 & & & & & 2 & 3,9 \\
\hline Total & 10 & 19,6 & 23 & 45,1 & 13 & 25,5 & 3 & 5,8 & 2 & 4,0 & 51 & 100,0 \\
\hline
\end{tabular}


Ao analisar a Tabela 2, verifica-se que a maior parte dos estudos foram conduzidos na Europa, com destaque para Inglaterra (país onde o SDQ foi originalmente publicado), Alemanha, Holanda e Suécia, totalizando mais da metade de todos os trabalhos realizados na Europa. No continente asiático foram conduzidos estudos referentes a aproximadamente $16 \%$ das publicações relacionadas às propriedades psicométricas do SDQ, dado que chama atenção principalmente pelos estudos conduzidos em países em desenvolvimento como Yemen, Paquistão e Sri Lanka. Outro aspecto merecedor de comentário é a ausência de estudos publicados, pelo menos nas bases de dados consultadas para esta revisão, na América do Sul. Entretanto, em um trabalho de revisão desenvolvido por Woerner, Becker e Rothenberger (2004) sobre as aplicações e avaliações do SDQ em países não europeus, são relatados estudos sobre as propriedades psicométricas do SDQ no Brasil, configurando-se como o único País da América do Sul com estudos dessa natureza.

No que diz respeito às propriedades psicométricas avaliadas e descritas na Tabela 2, a maior parte dos artigos realizou estudos de validade e fidedignidade, seguidos por pesquisas englobando também a padronização, ou seja, o estabelecimento de normas a partir de adaptações e/ou traduções.

\section{Validade}

Dos artigos que realizaram estudos de validação do SDQ, 29 (56,8\%) investigaram a validade de construto, $15(29,4 \%)$ avaliaram a validade do tipo convergente/concorrente, e 18 (35,3\%) analisaram a validade discriminativa. Cabe ressaltar que vários artigos analisaram mais de um tipo de validade.

Em relação à validade de construto, 18 artigos confirmaram a estrutura fatorial originalmente publicada por Goodman (2001), com uma solução de cinco fatores, enquanto três estudos não confirmaram essa estrutura e nove artigos a confirmaram com ressalva e/ou sugestão de melhoria. Dentre esses artigos, foram encontrados modelos com soluções fatoriais mais satisfatórias de três, quatro e até seis fatores. Outros recomendam que alguns itens sejam modificados e/ou movidos de uma escala para outra. De uma maneira geral, as escalas de Problemas de relacionamento com colegas e Problemas de conduta foram as que apresentaram os maiores problemas conceituais.

Em relação à validade convergente/concorrente, verificaram-se as seguintes variações nos índices de correlação: versão dos pais variando de 0,63 a 0,88, versão dos professores de 0,71 a 0,92, e versão de autorrelato com valores entre 0,69 e 0,75. Todas as correlações referem-se ao escore total de dificuldades.

Para as análises referentes à validade discriminativa, foram usados nas análises os procedimentos da Curva de Receiver Operating Characteristic (ROC) e diferença entre as médias dos escores (casos x não casos). Para a versão dos pais, a área sobre a curva Area Under the Curve (AUC) variou de 0,69 a 0,93; para a versão dos professores a AUC variou de 0,75 a 0,91; e para a versão de autorrelato, de 0,51 a 0,87.

\section{Utilização de padrão-ouro}

Dos 51 estudos analisados, 21 deles $(41,2 \%)$ utilizaram padrão-ouro, sendo os três mais utilizados o Development and Well-Being Assessment (DAWBA), o Child Behaviour Check List (CBCL) e suas versões, e a entrevista clínica realizada por psiquiatra.

\section{Correlação interavaliadores}

Dentre os estudos selecionados para esta revisão, nove deles $(17,6 \%)$ investigaram correlação interavaliadores, sendo encontrados os seguintes valores: Pais $x$ Professores, com valores para o escore total de dificuldades variando de 0,22 a 0,52; correlação Pais $x$ Autorrelato, com valores entre 0,39 a 0,60; correlação entre as versões Professores $X$ Autorrelato, de 0,27 a 0,45 . Um estudo analisou a correlação entre uma versão respondida pela mãe e a versão respondida pelo pai, com coeficiente de correlação de 0,71 (escore total).

\section{Fidedignidade}

Dos artigos incluídos nesta revisão, 37 (72,5\%) investigaram o índice de consistência interna do SDQ por meio do Alfa de Cronbach. Os valores do escore total de dificuldades, para a versão dos pais, variaram entre 0,59 a 0,88. Para a versão dos professores, o alfa de Cronbach variou de 0,60 a 0,88 (escore total), e para a 
versão de autorrelato entre 0,57 e 0,82. Em um dos estudos, foi analisada uma versão computadorizada do SDQ, cujo índice de consistência interna foi de 0,69 (escore total).

Foi verificado também que nove estudos (17,6\%) realizaram teste-reteste. A correlação entre o escore total de dificuldades variou entre 0,72 e 0,86 (versão dos pais), 0,55 e 0,90 (versão dos professores), 0,62 e 0,84 (versão de autorrelato), e de 0,83 para a versão computadorizada do SDQ. O intervalo para o teste-reteste variou de 19 dias até um ano.

\section{Estudos de padronização}

Verificou-se que 18 trabalhos envolveram estudos de padronização, sendo 10 (55,5\%) deles referentes ao estabelecimento de normas, 3 (16,7\%) relacionados a estudos de tradução de versões, e 5 (27,8\%) envolvendo os dois tipos de estudo.

Dentre os estudos analisados que realizaram o processo de tradução da versão original inglesa para seu respectivo idioma ( $n=8)$, estão Holanda, Finlândia, lêmen, Rússia, Paquistão, China, Suécia e Sri Lanka.

Em relação aos estudos que realizaram normatização, isto é, o estabelecimento de notas de corte $(n=15)$, verificam-se os seguintes valores de corte, todos referentes ao escore de dificuldades totais: para a versão dos pais foram identificados valores de corte variando de 11 a 19; para a versão respondida pelos professores, os pontos de corte variaram entre 18 e 19; e, para a versão de autorrelato, de 15 a 18 . O ponto de corte referente à norma britânica é de 17 para a versão dos pais e 16 para a versão dos professores.

\section{Principais limites apontados pelos autores}

pelos autores, destacam-se as seguintes características como pontos críticos: a) quanto às versões do SDQ, a maior parte dos estudos utilizou somente uma versão (versão dos pais), sendo recomendada a avaliação com mais informantes para ampliar a compreensão sobre os dados observados; b) quanto às características das amostras, verificou-se que alguns estudos trabalharam com um único tipo de amostra (da comunidade ou 624 amostra clínica), tendo sido apontado como necessária a realização de outros estudos com amostras mais representativas da população (como, por exemplo, diferentes áreas de moradia e diferentes etnias) e c) quanto às propriedades psicométricas, foi destacada pelos autores a necessidade de estudos sobre outras propriedades psicométricas não avaliadas em seus estudos. Outros limites apontados dizem respeito a estudos realizados sem a presença de um instrumento considerado como padrão-ouro e ao uso, em seus trabalhos, de notas de corte não padronizadas para o país onde foi conduzido o estudo.

\section{OStrengths and Difficulties Questionnaire no Brasil}

Recentemente, é notório o esforço da comunidade científica da área psicológica no Brasil em promover a melhoria na qualidade psicométrica dos instrumentos de avaliação psicológica utilizados (Primi, 2010). Nesse contexto, julga-se importante apresentar, ainda que de forma breve, os estudos brasileiros relacionados às propriedades psicométricas do SDQ.

Em estudo relatado por Woerner et al. (2004), foram descritos resumidamente os dados psicométricos sobre a validade e fidedignidade do SDQ no Brasil. Foram coletadas informações com pais e professores, tendo por amostra da comunidade 898 crianças e adolescentes de 7 a 14 anos, residentes na cidade de Campos do Jordão (SP). Para a amostra clínica, foram recrutados 87 pacientes de uma clínica em uma universidade na cidade de São Paulo, uma vez que na referida cidade não havia serviços de saúde mental infantil. Com relação à validade discriminativa, foram verificadas diferenças significativas $(p<0,001)$ entre a média do escore total da amostra da comunidade e clínica, para as versões do SDQ respondidas por pais e professores, embora não tenha sido encontrada diferença significativa para a versão de autorrelato. Quanto à validade de critério, os participantes da comunidade com SDQ positivo (presença de transtorno) foram avaliados pelo DAWBA, utilizado como padrão-ouro, verificando-se que 56\% da amostra apresentaram indicadores confirmados para algum diagnóstico de psicopatologia infantil, com base no Diagnostic and Statistical Manual of Mental Disorders, 4a edição - DSM-IV (Manual Diagnóstico e Estatístico de Transtornos Mentais - $4^{a}$ ed.). Também foram avaliados participantes da comunidade com SDQ negativo 
(ausência de transtorno), dos quais 15\% apresentaram indicadores confirmados para algum diagnóstico do DSM-IV, tendo como referência a avaliação feita pelo $\operatorname{DAWBA}\left(\chi^{2}=13,1 ; p<0,001\right)$.

Os estudos de fidedignidade do SDQ no Brasil foram definidos pelo cálculo do alfa de Cronbach e testereteste (amostra clínica de 17 participantes e intervalo médio de 20 dias entre as aplicações). Para o índice de consistência interna, o alfa de Cronbach apre-sentou, para as três versões utilizadas, valores próximos de 0,80 (valores para o escore total de dificuldades), sendo para o teste-reteste a correlação de 0,79.

Os dados indicam que o SDQ apresentou adequadas propriedades psicométricas aferidas para a população brasileira. Desse modo, configura-se como uma boa alternativa de uso, tendo se mostrado um instrumento útil na investigação de problemas de saúde mental de crianças e adolescentes.

Para verificar a aplicabilidade do SDQ no Brasil, realizou-se uma pesquisa não exaustiva na base de dados Lilacs, a qual abrange trabalhos da literatura latino-americana. Os termos pesquisados foram "Questionário de Capacidades e Dificuldades"e"Strengths and Difficulties Questionnaire", tendo sido identificados 11 estudos, sendo 10 deles desenvolvidos no Brasil. Por meio de leitura de seus conteúdos, verificou-se que dos 10 trabalhos desenvolvidos no Brasil, cinco relacionavam problemas de saúde mental (identificados pelo SDQ) a outras variáveis, como, por exemplo, peso ao nascer (Gallo et al., 2011), tabagismo (Menezes et al., 2011), bullying (Moura, Cruz\& Quevedo, 2011), asma (Alvim et al., 2008) e variáveis do contexto familiar (Ferrioli, Marturano \& Puntel, 2007). Quatro trabalhos utilizaram o SDQ para fins específicos, a saber: na identificação de problemas de atenção e hiperatividade (Anselmi et al., 2010), na identificação de características comportamentais de crianças leitoras (Stivanin, Scheuer \& Assumpção Júnior, 2008), na investigação de desordens psiquiátricas infantis (Cury \& Golfeto, 2003) e na avaliação do comportamento social na escola (Saud \& Tonelotto, 2005), além de um estudo que abordou aspectos conceituais relativos ao SDQ (Fleitlich et al., 2000).

Apesar de não estarem indexados na base de dados consultada, três outros trabalhos que utilizaram o SDQ também podem ser citados. Dois trabalhos abordaram a prevalência de desordens psiquiátricas, um deles em uma comunidade rural afro-brasileira no Nordeste do país (Goodman et al., 2005) e outro no Sudeste do País (Fleitlich-Bilyk \& Goodman, 2004). O terceiro artigo utilizou o SDQ na avaliação de problemas de saúde mental relacionados a fatores socioeconômicos e perinatais, em estudo conduzido no Nordeste (Rodriguez, da Silva, Bettiol, Barbieri \& Rona, 2011).

\section{Considerações Finais}

Com base na análise dos estudos, verificou-se que $82,3 \%$ deles $(n=42$ ) relataram qualidades psicométricas satisfatórias para o SDQ, com índices positivos de validade e fidedignidade, destacando a sua adequação e aplicabilidade como rastreador de problemas de saúde mental de crianças e adolescentes, inclusive no Brasil. Esses estudos confirmaram a estrutura original de cinco fatores e demonstraram índices psicométricos satisfatórios em diferentes contextos e populações, evidenciando seu alcance transcultural, o que constitui importante parâmetro de aplicabilidade. Destaca-se ainda o grande número de participantes dos estudos, uma vez que 20,0\% deles incluíram mais de 5 mil sujeitos em sua amostra, quantidade bastante expressiva para estudos de avaliação de propriedades psicométricas. O uso das três versões do SDQ em boa parte dos artigos analisados (20,0\%) também merece ser enfatizado, dada a relevância de informações coletadas com base em múltiplos informantes ao se avaliar a saúde mental infanto-juvenil.

Outro aspecto interessante diz respeito ao ano de publicação dos estudos analisados. Verificou-se que 53\% deles ocorreram nos últimos cinco anos, indicando que, apesar da publicação original do SDQ ter ocorrido em 1997, o instrumento tem sido recentemente objeto de estudos psicométricos, caracterizando assim o reconhecimento de suas potencialidades para novas pesquisas.

Considerando a análise da literatura revisada, em especial a abrangência dos estudos conduzidos em diferentes países, incluindo o Brasil, conclui-se que o SDQ pode ser considerado um instrumento útil para a avaliação de indicadores de saúde mental infantil. Por se tratar de um instrumento de uso livre, fácil acesso, rápida aplicação e simples correção, ele constitui uma boa alternativa para a avaliação da saúde mental de 
crianças e adolescentes. Vale ressaltar que, apesar de o SDQ estar aferido para a população brasileira, com dados psicométricos positivos, sugere-se a realização de novos estudos sobre suas propriedades psicométricas em amostras mais representativas e diversificadas da população. Nesse sentido, espera-se que este trabalho seja o ponto de partida para alavancar novas pesquisas, no sentido de contribuir para o avanço da avaliação psicológica no Brasil, onde o uso de medidas psicometricamente adequadas se reveste de extrema importância na atualidade.

\section{Referências}

Achenbach, T. M. (1991). Manual for the Child Behaviour Checklist/4-18 and 1991 Profile. Burlington, VT: University of Vermont.

Almaqrami, M. H., \& Shuwail, A. Y. (2004). Validity of the self-report version of the strengths and difficulties questionnaire in Yemen. Saudi Medical Journal, 25 (5), 592-601.

Alvim, C. G., Ricas, J., Camargos, P. A. M., Lasmar, L. M. B. L. F., Andrade, C. R., \& Ibiapina, C. C. (2008). Prevalência de transtornos emocionais e comportamentais em adolescentes com asma. Jornal Brasileiro de Pneumologia, 34 (4), 196-204.

Alyahri, A., \& Goodman, R. (2006). Validation of the Arabic Strengths and Difficulties Questionnaire and the Development and Well-Being Assessment. Eastearn Mediterranean Health Journal, 12 (Suppl. 2), S138-146.

Anselmi, L., Menezes, A. M. B., Barros, F. C., Hallal, P. C., Araújo, C. L., Domingues, M. R., et al. (2010). Determinantes precoces de problemas de atenção e hiperatividade na adolescência: a visita de 11 anos da coorte de nascimentos de Pelotas, Rio Grande do Sul, Brasil, 1993. Cadernos de Saúde Pública, 26 (10), 1954-1962.

Becker, A., Hagenberg, N., Roessner, V., Woerner, W., \& Rothenberger, A. (2004). Evaluation of the self-reported SDQ in a clinical setting: do self-reports tell us more than ratings by adult informants? European Child \& Adolescent Psychiatry, 13 (Suppl. 2), II17-24.

Becker, A., Steinhausen, H. C., Baldursson, G., Dalsgaard, S., Lorenzo, M. J., Ralston, S. J., et al. (2006). Psychopathological screening of children with ADHD: strengths and difficulties questionnaire in a pan-European study. European Child \& Adolescent Psychiatry, 15 (Suppl. 1), 56-62.

Becker, A., Woerner, W., Hasselhorn, M., Banaschewski, T., \& Rothenberger, A. (2004). Validation of the parent and teacher SDQ in a clinical sample. European Child \& Adolescent Psychiatry, 13 (Suppl. 2), 11-16.

Blom, E. H., Larsson, J. O., Serlachius, E., \& Ingvar, M. (2010). The differentiation between depressive and anxious adolescent females and controls by behavioural self-rating scales. Journal of Affective Disorders, 122 (3), 232-240.
Bourdon, K. H., Goodman, R., Rae, D. S., Simpson, G., \& Koretz, D. S. (2005). The Strengths and Difficulties Questionnaire: U.S. normative data and psychometric properties. Journal of the American Academy of Child \& Adolescent Psychiatry, 44 (6), 557-564.

Crone, M. R., Vogels, A. G., Hoekstra, F., Treffers, P. D., \& Reijneveld, S. A. (2008). A comparison of four scoring methods based on the parent-rated Strengths and Difficulties Questionnaire as used in the Dutch preventive child health care system. BMC Public Health, 8, 106.

Cury, C. R., \& Golfeto, J. H. (2003). Questionário de capacidades e dificuldades (SDQ): um estudo em escolares de Ribeirão Preto. Revista Brasileira de Psiquiatria, 25 (3), 139-145

Di Riso, D., Salcuni, S., Chessa, D., Raudino, A., Lis, A., \& Altoè, G. (2010). The Strengths and Difficulties Questionnaire (SDQ): early evidence of its reliability and validity in a community sample of Italian children. Personality and Individual Differences, 49 (6), 570-575.

Dickey, W. C., \& Blumberg, S. J. (2004). Revisiting the factor structure of the strengths and difficulties questionnaire: United States, 2001. Journal of the American Academy of Child \& Adolescent Psychiatry, 43 (9), 1159-1167.

Du, Y., Kou, J., \& Coghill, D. (2008). The validity, reliability and normative scores of the parent, teacher and self report versions of the Strengths and Difficulties Questionnaire in China. Child and Adolescent Psychiatry and Mental Health, 2 (1), 8.

Ferrioli, S. H. T, Marturano, E. M., \& Puntel, L. P. (2007). Contexto familiar e problemas de saúde mental infantil no Programa de Saúde da Família. Revista de Saúde Pública, 41 (2), 251-259.

Fleitlich, B., Cortázar, P. G., \& Goodman, R. (2000). Questionário de capacidades e dificuldades (SDQ). Infanto-Revista de Neuropsiquiatria da Infância e Adolescência, 8 (1), 44-50.

Fleitlich-Bilyk, B., \& Goodman, R. (2004). Prevalence of child and adolescent psychiatric disorders in Southeast Brazil. Journal of the American Academy of Child \& Adolescent Psychiatry, 43 (6), 727-734.

Gallo, E. A. G., Anselmi, L., Dumith, S. C., Scazufca, M., Menezes, A. M. B, Hallal, P. C., et al. (2011). Tamanho ao nascer e problemas de saúde mental aos 11 anos em uma coorte brasileira de nascimentos. Cadernos de Saúde Pública, 27 (8), 1622-1632.

Goodman, R. (1997). The Strengths and Difficulties Questionnaire: a research note. Journal of Child Psychology and Psychiatry, 38 (5), 581-586.

Goodman, R. (1999). The extended version of the Strengths and Difficulties Questionnaire as a guide to child psychiatric caseness and consequent burden. Journal of Child Psychology and Psychiatry, 40 (5), 791-799.

Goodman, R. (2001). Psychometric properties of the strengths and difficulties questionnaire. Journal of the American Academy of Child \&Adolescent Psychiatry, 40 (11), 1337-1345.

Goodman, R., Neves dos Santos, D. N., Nunes, A. P. R., de Miranda, D. P., Fleitlich-Bilyk, B., Almeida Filho, N., et al. (2005). The Ilha de Maré study: a survey of child mental 
health problems in a predominantly African-Brazilian rural community. Social Psychiatry and Psychiatric Epidemiology, 40 (1), 11-17.

Goodman, A., \& Goodman, R. (2009). Strengths and difficulties questionnaire as a dimensional measure of child mental health. Journal of the American Academy of Child \& Adolescent Psychiatry, 48 (4), 400-403

Goodman, A., Lamping, D. L., \& Ploubidis, G. B. (2010). When to use broader internalising and externalising subscales instead of the hypothesised five subscales on the Strengths and Difficulties Questionnaire (SDQ): data from British parents, teachers and children. Journal of Abnormal Child Psychology, 38 (8), 1179-1191

Goodman, R., Meltzer, H., \& Bailey, V. (1998). The Strengths and Difficulties Questionnaire: a pilot study on the validity of the self-report version. European Child \& Adolescent Psychiatry, 7 (3), 125-130.

Goodman, R., \& Scott, S. (1999). Comparing the Strengths and Difficulties Questionnaire and the Child Behavior Checklist: is small beautiful? Journal of Abnormal Child Psychology, 27 (1), 17-24.

Hagquist, C. (2007). The psychometric properties of the self-reported SDQ: an analysis of Swedish data based on the Rasch model. Personality and Individual Differences, 43 (5), 1289-1301.

Hawes, D. J., \& Dadds, M. R. (2004). Australian data and psychometric properties of the Strengths and Difficulties Questionnaire. Australian and New Zealand Journal of Psychiatry, 38 (8), 644-651.

Hill, C. R., \& Hughes, J. N. (2007). An Examination of the Convergent and Discriminant Validity of the Strengths and Difficulties Questionnaire. School Psychology Quarterly, 22 (3), 380-406.

Hintermair, M. (2007). Prevalence of socioemotional problems in deaf and hard of hearing children in Germany. American Annals of the Deaf, 152 (3), 320-330.

Hysing, M., Elgen, I., Gillberg, C., Lie, S. A., \& Lundervold, A. J. (2007). Chronic physical illness and mental health in children. Results from a large-scale population study. Journal of Child Psychology and Psychiatry, 48 (8), 785-792.

Janssens, A., \& Deboutte, D. (2009). Screening for psychopathology in child welfare: the Strengths and Difficulties Questionnaire (SDQ) compared with the Achenbach System of Empirically Based Assessment (ASEBA). European Child \& Adolescent Psychiatry, 18 (11), 691-700.

Kaptein, S., Jansen, D. E., Vogels, A. G., \& Reijneveld, S. A. (2008). Mental health problems in children with intellectual disability: use of the Strengths and Difficulties Questionnaire. Journal of Intellectual Disability Research, 52 (Pt 2), 125-131.

Kashala, E., Elgen, I., Sommerfelt, K., \& Tylleskar, T. (2005). Teacher ratings of mental health among school children in Kinshasa, Democratic Republic of Congo. European Child \& Adolescent Psychiatry, 14 (4), 208-215.

Klasen, H., Woerner, W., Wolke, D., Meyer, R., Overmeyer, S., Kaschnitz, W., et al. (2000). Comparing the German versions of the Strengths and Difficulties Questionnaire
(SDQ-Deu) and the Child Behavior Checklist. European Child \& Adolescent Psychiatry, 9 (4), 271-276.

Koskelainen, M., Sourander, A., \& Vauras, M. (2001). Selfreported strengths and difficulties in a community sample of Finnish adolescents. European Child \& Adolescent Psychiatry, 10 (3), 180-185.

Lai, K. Y. C, Luk, E. S. L., Leung, P. W. L., Wong, A. S. Y., Law, L, \& Ho, K. (2009). Validation of the Chinese version of the strengths and difficulties questionnaire in Hong Kong. Social Psychiatry and Psychiatric Epidemiology, 45(12), 1179-1186.

Lukumar, P., Wijewardana, K., Hermansson, J., \& Lindmark, G. (2008). Validity and reliability of Tamil version of Strengths and Difficulties Questionnaire self-report. Ceylon Medical Journal, 53 (2), 48-52.

Lundh, L. G., Wangby-Lundh, M., \& Bjarehed, J. (2008). Selfreported emotional and behavioral problems in Swedish 14 to 15-year-old adolescents: a study with the self-report version of the Strengths and Difficulties Questionnaire. Scandinavian Journal of Psychology, 49 (6), 523-532.

Malmberg, M., Rydell, A. M., \& Smedje, H. (2003). Validity of the Swedish version of the Strengths and Difficulties Questionnaire (SDQ-Swe). Nordic Journal of Psychiatry, 57 (5), 357-363.

Matsuishi, T., Nagano, M., Araki, Y., Tanaka, Y., Iwasaki, M., Yamashita, Y., et al. (2008). Scale properties of the Japanese version of the Strengths and Difficulties Questionnaire (SDQ): a study of infant and school children in community samples. Brain Development, 30 (6), 410-415.

Mellor, D. (2004). Furthering the use of the strengths and difficulties questionnaire: reliability with younger child respondents. Psychological Assessment, 16 (4), 396-401.

Menezes, A. M. B, Dumith, S. C., Martínez-Mesa, J., Silva, A. E. R., Cascaes, A. M., Domínguez, G. G., et al. (2011). Problemas de saúde mental e tabagismo em adolescentes do sul do Brasil. Revista de Saúde Publica, 45 (4), 700-705.

Mojtabai, R. (2006). Serious emotional and behavioral problems and mental health contacts in American and British children and adolescents. Journal of the American Academy of Child \& Adolescent Psychiatry, 45 (10), 1215-1223.

Moura, D. R., Cruz, A. C. N., \& Quevedo, L. A. (2011). Prevalência e características de escolares vítimas de bullying. Jornal de Pediatria, 87 (1), 19-23.

Muris, P., Meesters, C., Eijkelenboom, A., \& Vincken, M. (2004). The self-report version of the Strengths and Difficulties Questionnaire: its psychometric properties in 8- to 13-year-old non-clinical children. British Journal of Clinical Psychology, 43 (Pt 4), 437-448.

Muris, P., Meesters, C., \& van den Berg, F. (2003). The Strengths and Difficulties Questionnaire (SDQ): further evidence for its reliability and validity in a community sample of Dutch children and adolescents. European Child \& Adolescent Psychiatry, 12 (1), 1-8.

Noronha, A. P. P., Primi, R., \& Alchieri, J. C. (2004). Parâmetros psicométricos: uma análise de testes psicológicos comercializados no Brasil. Psicologia Ciência eProfissão, 24, 88-89. 
Norwood, S. (2007). Validity of self-reports of psychopathology from children of 4-11 years of age. Vulnerable Children and Youth Studies, 2 (2), 89-99.

Palmieri, P. A., \& Smith, G. C. (2007). Examining the structural validity of the Strengths and Difficulties Questionnaire (SDQ) in a U.S. sample of custodial grandmothers. Psychological Assessment, 19 (2), 189-198.

Primi, R. (2010). Avaliação psicológica no Brasil:fundamentos, situação atual e direções para o futuro. Psicologia: Teoriae Pesquisa, 26, 25-35.

Rodriguez, J. D. M., da Silva, A. A. M., Bettiol, H., Barbieri, M. A., \& Rona, R. (2011). The impact of perinatal and socioeconomic factors on mental health problems of children from a poor Brazilian city: a longitudinal study. Social Psychiatry and Psychiatric Epidemiology, 46 (5), 381-91.

Ronning, J. A., Handegaard, B. H., Sourander, A., \& Morch, W. T. (2004). The Strengths and Difficulties Self-Report Questionnaire as a screening instrument in Norwegian community samples. European Child \& Adolescent Psychiatry, 13 (2), 73-82.

Rothenberger, A., Becker, A., Erhart, M., Wille, N., \& RavensSieberer, U. (2008). Psychometric properties of the parent strengths and difficulties questionnaire in the general population of German children and adolescents: results of the BELLA study. European Child \&Adolescent Psychiatry, 17 (Suppl. 1), 99-105.

Ruchkin, V., Koposov, R., \& Schwab-Stone, M. (2007). The Strength and Difficulties Questionnaire: scale validation with Russian adolescents. Journal of Clinical Psychology, 63 (9), 861-869.

Rutter, M. (1967). A children's behaviour questionnaire for completion by teachers: preliminary findings. Journal of Child Psychology and Psychiatric, 8 (1), 1-11.

Samad, L., Hollis, C., Prince, M., \& Goodman, R. (2005). Child and adolescent psychopathology in a developing country: testing the validity of the strengths and difficulties questionnaire (Urdu version). International Journal of Methods in Psychiatric Research, 14 (3), 158-166.

Saud, L. F., \& Tonelotto, J. M. F.(2005). Comportamento social na escola: diferenças entre gênero e séries. Psicologia Escolar Educacional, 9 (1), 47-57.

Shojaei, T., Wazana, A., Pitrou, I., \& Kovess, V. (2009). The strengths and difficulties questionnaire: validation study in French school-aged children and cross-cultural comparisons. Social Psychiatry and Psychiatric Epidemiology, 44 (9), 740-747.
Smedje, H., Broman, J. E., Hetta, J., \& von Knorring, A. L. (1999). Psychometric properties of a Swedish version of the "Strengths and Difficulties Questionnaire". European Child \& Adolescent Psychiatry, 8 (2), 63-70.

Stivanin, L., Scheuer, C. I., \& Assumpção Júnior, F. B. (2008). SDQ (Strengths and Difficulties Questionnaire): identificação de características comportamentais de crianças leitoras. Psicologia: Teoria e Pesquisa 24 (4), 407-413.

Truman, J., Robinson, K., Evans, A. L., Smith, D., Cunningham, L., Millward, R., et al. (2003). The Strengths and Difficulties Questionnaire: a pilot study of a new computer version of the self-report scale. European Child \& Adolescent Psychiatry, 12 (1), 9-14.

Van Roy, B., Veenstra, M., \& Clench-Aas, J. (2008). Construct validity of the five-factor Strengths and Difficulties Questionnaire (SDQ) in pre-, early, and late adolescence. Journal of Child Psychology and Psychiatry, 49 (12), 1304-1312.

Van Widenfelt, B. M., Goedhart, A. W., Treffers, P. D., \& Goodman, R. (2003). Dutch version of the Strengths and Difficulties Questionnaire (SDQ). European Child \& Adolescent Psychiatry, 12 (6), 281-289.

Vogels, A. G., Crone, M. R., Hoekstra, F., \& Reijneveld, S. A. (2009). Comparing three short questionnaires to detect psychosocial dysfunction among primary school children: a randomized method. BMC Public Health, 9, 489.

Vostanis, P. (2006). Strenghts and Difficulties Questionnaire: research and clinical aplications. Current Opinion Psychiatric, 19 (4), 367-372.

Woerner, W., Becker, A., \& Rothenberger, A. (2004). Normative data and scale properties of the German parent SDQ. European Child \& Adolescent Psychiatry, 13 (Suppl. 2), II3-10.

Woerner, W., Fleitlich-Bilyk, B., Martinussen, R., Fletcher, J., Cucchiaro, G., Dalgalarrondo, P., et al. (2004). The Strengths and Difficulties Questionnaire overseas: evaluations and applications of the SDQ beyond Europe. European Child \& Adolescent Psychiatry, 13 (Suppl. 2), ||47-54.

Yao, S., Zhang, C., Zhu, X., Jing, X., McWhinnie, C. M., \& Abela, J. R. (2009). Measuring adolescent psychopathology: psychometric properties of the self-report strengths and difficulties questionnaire in a sample of Chinese adolescents. Journal of Adolescent Health, 45 (1), 55-62.

Recebido em: 25/11/2010

Versão final em: 27/1/2012

Aprovado em: 28/3/2012 
ANEXO

Estudos analisados, país de realização dos estudos e propriedades psicométricas avaliadas ( $n=51$ )

\begin{tabular}{|c|c|c|}
\hline Artigo (Autor/Ano) & País de estudo & Propriedade psicométrica analisada \\
\hline Almaqrami e Shuwail (2004) & Yemen & Validade e padronização \\
\hline Alyahri e Goodman (2006) & Yemen & Validade \\
\hline Becker, Hagenberg, Roessner, Woerner e Rothenberger (2004) & Alemanha & Validade e fidedignidade \\
\hline Becker et al. (2006) & Vários países da Europa & Validade e fidedignidade \\
\hline Becker, Woerner, Hasselhorn, Banaschewski e Rothenberger (2004) & Alemanha & Validade e fidedignidade \\
\hline Blom, Larsson, Serlachius e Ingvar (2010) & Suécia & Validade \\
\hline Bourdon, Goodman, Rae, Simpson e Koretz (2005) & Estados Unidos & Fidedignidade e padronização \\
\hline Crone, Vogels, Hoekstra, Treffers e Reijneveld (2008) & Holanda & Validade \\
\hline Di Riso et al. (2010) & Itália & Validade e fidedignidade \\
\hline Dickey e Blumberg (2004) & Estados Unidos & Validade \\
\hline Du, Kou e Coghill (2008) & China & Validade, fidedignidade e padronização \\
\hline Goodman (1997) & Inglaterra & Validade \\
\hline Goodman (1999) & Inglaterra & Validade e fidedignidade \\
\hline Goodman (2001) & Inglaterra & Validade e fidedignidade \\
\hline A. Goodman e R. Goodman (2009) & Inglaterra & Validade \\
\hline Goodman, Lamping e Ploubidis (2010) & Inglaterra & Validade \\
\hline Goodman, Meltzer e Bailey (1998) & Inglaterra & Validade e fidedignidade \\
\hline Goodman e Scott (1999) & Inglaterra & Validade \\
\hline Hagquist (2007) & Suécia & Validade e fidedignidade \\
\hline Hawes e Dadds (2004) & Austrália & Validade, fidedignidade e padronização \\
\hline Hill e Hughes (2007) & Estados Unidos & Validade e fidedignidade \\
\hline Hintermair (2007) & Alemanha & Validade e fidedignidade \\
\hline Hysing, Elgen, Gillberg, Lie e Lundervold (2007) & Noruega & Validade \\
\hline Janssens e Deboutte (2009) & Bélgica & Validade e fidedignidade \\
\hline Kaptein, Jansen, Vogels e Reijneveld (2008) & Holanda & Validade e fidedignidade \\
\hline Kashala, Elgen, Sommerfelt e Tylleskar (2005) & Congo & Validade, fidedignidade e padronização \\
\hline Klasen et al. (2000) & Alemanha & Validade \\
\hline Koskelainen, Sourander e Vauras (2001) & Finlândia & Validade, fidedignidade e padronização \\
\hline Lai et al. (2009) & China & Validade, fidedignidade e padronização \\
\hline Lukumar, Wijewardana, Hermansson e Lindmark (2008) & Sri Lanka & Validade, fidedignidade e padronização \\
\hline Lundh, Wangby-Lundh e Bjarehed (2008) & Suécia & Validade, fidedignidade e padronização \\
\hline Malmberg, Rydell e Smedje (2003) & Suécia & Validade e fidedignidade \\
\hline Matsuishi et al. (2008) & Japão & Validade, fidedignidade e padronização \\
\hline Mellor (2004) & Austrália & Validade e fidedignidade \\
\hline Mojtabai (2006) & Estados Unidos e Inglaterra & Validade e fidedignidade \\
\hline Muris, Meesters, Eijkelenboom e Vincken (2004) & Holanda & Validade e fidedignidade \\
\hline Muris, Meesters e van den Berg (2003) & Holanda & Validade e fidedignidade \\
\hline Norwood (2007) & Inglaterra & Validade e fidedignidade \\
\hline Palmieri e Smith (2007) & Estados Unidos & Validade e fidedignidade \\
\hline Ronning, Handegaard, Sourander e Morch (2004) & Noruega & Validade, fidedignidade e padronização \\
\hline Rothenberger, Becker, Erhart, Wille e Ravens-Sieberer (2008) & Alemanha & Validade e fidedignidade \\
\hline Ruchkin, Koposov e Schwab-Stone (2007) & Rússia & Validade e padronização \\
\hline Samad, Hollis, Prince e Goodman (2005) & Paquistão & Validade, fidedignidade e padronização \\
\hline Shojaei, Wazana, Pitrou e Kovess (2009) & França & Fidedignidade e padronização \\
\hline Smedje, Broman, Hetta e von Knorring (1999) & Suécia & Validade, fidedignidade e padronização \\
\hline Truman et al. (2003) & Inglaterra & Validade e fidedignidade \\
\hline Van Roy, Veenstra e Clench-Aas (2008) & Noruega & Validade e fidedignidade \\
\hline Van Widenfelt, Goedhart, Treffers e Goodman (2003) & Holanda & Validade, fidedignidade e padronização \\
\hline Vogels, Crone, Hoekstra e Reijneveld (2009) & Holanda & Validade e fidedignidade \\
\hline Woerner, Becker e Rothenberger (2004) & Alemanha & Validade, fidedignidade e padronização \\
\hline Yao et al. (2009) & China & Validade e fidedignidade \\
\hline
\end{tabular}

